\title{
BRONQUIOLITE AGUDA COMO CONDIÇÃO SENSÍVEL A ATENÇÃO PRIMÁRIA, EM UMA CIDADE NO SUL DO BRASIL
}

\section{ACUTE BRONQUIOLITIS AS AN AMBULATORY CARE SENSITIVE CONDITION, IN A CITY IN THE SOUTH OF BRAZIL}

Letícia Oliveira de Menezes, Carolina Reinbrecht Bernini, Marcos Daniel da Silva, Thais Leite Secchi, Sandro Schreiber de Oliveira

\begin{abstract}
RESUMO
ABronquiolite, que ocorre frequentemente em lactentes jovens, com predomínio nos meses de inverno é sensível a atenção primária à Saúde (APS) pela portaria $n^{0} 221$ do dia 17 de abril de 2008 do Ministério da Saúde, porém ocupa um grande contingente hospitalar. O objetivo do estudo foi avaliar os índices de internação hospitalar referentes à bronquiolite em crianças de faixa etária de 0-9 anos, no período de 2012/2013, em Pelotas, avaliando a qualidade da atenção básica e seus efeitos no processo de internação. Constatou-se que ocorreram 323 internações, sendo 174 em 2012 e 149 em 2013. A faixa etária mais prevalente foi em crianças menores de 1 ano $(83,59 \%)$, seguida pela faixa de 1 a 4 anos (15,17\%), e de 5 a 9 anos (1,23\%). A doença é autolimitada e é prevenível por medidas simples de higiene, assim é possível depreender que o número de hospitalizações por bronquiolite pode estar relacionada à carência de ações de prevenção efetivas nos serviços de APS.
\end{abstract}

Descritores: atenção primária em saúde; internação hospitalar; sistemas de saúde; bronquiolite;

\section{ABSTRACT}

The bronchiolitis, que often occurs in young infants, with predominance In the winter months . And Sensitive Primary Health Care (PHC) by decree No. 221 of 17 April 2008 of the Ministry of Health, however occupies hum large contingent hospital . OS was observed hospitalization rates related to bronchiolitis in Age of Children 0-9 years, no period 2012/2013, in Pelotas, assessing the Quality of Primary and ITS effects on Admission Process. -If Found que were 323 hospitalizations, being 174 in 2012 and 149 in 2013. More prevalent age group was in Children under 1 year ( $83.59 \%$ ) , after $1-4$ years $(15.17 \%)$, and $5-9$ year $(1.23 \%)$. The Health and selflimited and and preventable BY simple hygiene measures it is possible to infer that the number of hospitalizations for bronchiolitis may be related to the lack of effective actions Prevention nos PHC services .

Descriptors: primary health care; hospitalizations; health system; acute bronchiolitis;
Leticia Oliveira de Menezes

Formação Profissional: Administradora de Sistemas e Serviços de Saúde

Filiação Institucional: Universidade Católica de Pelotas Endereço para correspondência (rua, número, bairro, CEP cidade, UF, pais): Rua Gonçalves Chaves 3657/304, Centro, Pelotas, RS. CEP 96015-560

Carolina Reinbrecht Bernini

Formação Profissional: Estudante Medicina Filiação Institucional: Universidade Católica de Pelotas

\section{Marcos Daniel da Silva}

Formação Profissional: Estudante Medicina Filiação Institucional: Universidade Católica de Pelotas

Thais Leite Secchi

Formação Profissional: Estudante Medicina Filiação Institucional: Universidade Católica de Pelotas

Sandro Schreiber de Oliveira

Formação Profissional: Médico

Filiação Institucional: Universidade Católica de Pelotas Universidade Federal do Rio Grande 


\section{INTRODUÇÃO}

A portaria n²21, de 17 de abril de 2008, dispõe uma lista de Condições Sensiveis à Atenção Primária em Saúde (CSAP), que são agravos à saúde cuja morbimortalidade pode ser reduzida através de uma atenção primária eficaz ${ }^{(1,2)}$ As taxas de internação associadas a essas condições são usadas como instrumento avaliador da qualidade da atenção básica prestada pelo Sistema Único de Saúde, pois indicam deficiências na cobertura e no acesso de serviços pela população na esfera primária. Entre as condições sensiveis à atenção primária referidas pela portaria supracitada encontra-se a bronquiolite aguda. ${ }^{(3)}$

Bronquiolite é definida como uma síndrome respiratória aguda que ocorre frequentemente em lactentes jovens, caracterizada por inflamação e infecção do trato respiratório inferior, levando a obstruções e atelectasias ${ }^{(4,5)}$. A etiologia é predominantemente viral, mas em alguns casos pode ser bacteriana( ${ }^{(4)}$. 0 principal agente etiológico é o vírus sincicial respiratório, que existe em todo o mundo e eventualmente causa surtos sazonais, principalmente nos meses de outono e inverno, ou nos meses de chuva nos locais de clima tropical(6). Em crianças saudáveis é uma doença tipicamente autolimitada e com mortalidade baixa $(<1 \%)$, no entanto, constitui uma das principais causas de hospitalização em lactentes e crianças durante os primeiros cinco anos de vida ${ }^{(4,5)}$. Entre os fatores de risco para severidade e complicações da bronquiolite aguda encontram-se: prematuridade (IG<37 semanas), idade $<12$ semanas, doença pulmonar crônica (particularmente displasia broncopulmonar), doença cardíaca congênita, doença neurológica, imunodeficiência4 ${ }^{4}$. Outros fatores também podem contribuir para um quadro mais severo de bronquiolite, como fumo passivo, aglomeração, permanência em creches, ser gemelar, ter irmãos mais velhos e estar em altitude elevada $(>2500 \text { metros })^{(7)}$.

O manejo ambulatorial pode estar indicado quando a criança encontra-se adequadamente hidratada e sem sinais de esforço respiratório ou necessidade de suplementação de oxigênio. Nesses casos é importante promover educação em saúde aos pais e orientação quanto ao tratamento sintomático e de suporte, como desobstrução nasal, hidratação e monitoramento da progressão da doença ${ }^{(7)}$. A resolução do quadro costuma ocorrer em torno de 8-15 dias, apenas 10\% dos casos costumam apresentar sintomas por até 4 semanas ${ }^{(5,6,7)}$.

A hospitalização é indicada para crianças em mau estado geral, letargia, desidratação, recusa alimentar, com esforço respiratório moderado a severo (batimento de asa de nariz, tiragem subcostal, intercostal ou de fúrcula, gemência, frequência respiratória $>70$, dispneia, cianose), apneia, hipoxemia e situações onde os pais ou responsáveis não tem condições de oferecer cuidado domiciliar adequado ${ }^{4}$. Em crianças $>6$ meses, hospitalizadas, o tempo médio de internação é de 3-4 dias, tempo que pode ser maior em crianças menores ou com comorbidades ${ }^{(4,7)}$.

Algumas estratégias simples para reduzir o risco e a morbidade da bronquiolite incluem higiene das mãos, minimização de exposição passiva ao cigarro e abstenção do contato com indivíduos com infeç̧ões do trato respiratório. Uma opção é a imunoprofilaxia com palivizumabe (anticorpo monoclonal contra o vírus sincicial respiratório), que reduz 0 risco de hospitalização entre crianças prematuras, com displasia broncopulmonar ou doença cardíaca congênita ${ }^{(7)}$. Além disso, a vacinação contra outros agentes virais da bronquiolite aguda, como o vírus influenza, contribui para a redução dos casos dessa doença.
O DATASUS, base de dados regida por meio do decreto $\mathrm{N}^{0} 7.530$ de 21 de julho de 2011, tem a bronquiolite como doença passível de notificação. Neste, a bronquiolite está disposta em: J21: Bronquiolite aguda; J21.0: Bronquiolite aguda devido ao vírus sincicial respiratório; J21.8: Bronquiolite aguda devido a outro microrganismo espećíico; J21.9: Bronquite aguda NE (não-especificada). No entanto, a portaria SAS/MS n 221, de 17 de abril de 2008, que dispõe sobre as Condições Sensiveis à Atenção Primária em Saúde, considera todas essas classificações de forma geral como Bronquiolite, atribuindo a elas apenas o código CID J21 (os códigos utilizados em sua alimentação/manutenção no que se refere às doenças notificadas são os mesmos determinados pelo $\mathrm{CID}-10^{7}$ elaborado pela Organização Mundial da Saúde).

Neste contexto, sendo a bronquiolite, em geral, uma doença autolimitada e com possibilidade de prevenção por medidas simples de higiene e imunizações, parece inadequado que ela figure entre as maiores causas de internações em crianças. Esta situação sugere uma atenção primária deficitária e indica a necessidade de qualificação do cuidado básico em saúde. Portanto, realizar ações de monitoramento e avaliação por meio de ferramentas como o DATASUS pode ser uma estratégia para repensar e qualificar a participação da atenção básica no processo saúde-doença dos indivíduos assistidos por esses serviços.

Neste sentido, este estudo propõe-se a analisar as internações por causas sensiveis à atenção primária, em especial a bronquiolite aguda, na população de 0-9 anos, no período de 2012-2013, no município de Pelotas, levando em conta a influência da qualidade dos serviços de atenção básica em saúde.

\section{METODOLOGIA}

Trata-se de um estudo transversal, de base secundária que tem por objetivo analisar as internações hospitalares ocasionadas por bronquiolite aguda no município de Pelotas. Os dados para esse estudo foram obtidos através da base de dados DATASUS e foram coletados em setembro de 2014. Foram considerados os dados referentes ao período de 2012/2013. Todos os dados disponíveis para o período foram incluídos no estudo, totalizando 326 casos. As variáveis selecionadas para esse estudo foram: Causa da internação (de acordo com o CID-10) e a idade do paciente.

\section{RESULTADOS}


A Tabela 1 dispõe sobre as internações decorridas do diagnóstico de bronquiolite e suas subdivisões no município de Pelotas-RS por faixa etária. Através de sua análise, é possível aponta que a maior concentração de internações por bronquiolite aguda (CID J21) se deu nos indivíduos menores de 1 ano, sendo a subdivisão mais prevalente a Bronquiolite aguda devido a outro microrganismo específico (CID J21.8).

Na tabela 1, observa-se ainda a baixa prevalência de internações por bronquiolites em indivíduos com mais de 9 anos de idade, portanto, esse estudo dará ênfase aos indivíduos distribuídos entre 0 a 9 anos. Pode-se notar que entre os indivíduos de 0 a 9 anos, a maior parte das internações por bronquiolite tiveram como sujeitos indivíduos menores de 1 ano, correspondendo a 83,59\% da amostra.

Tabela 1 - Internações devido à bronquiolite aguda e suas diferentes etiologias, distribuídas por faixa etária, por dois anos no município de Pelotas, RS

\begin{tabular}{lccccc}
\hline Diagnóstico pelo CID10 & $<$ 1ano & $1-4$ anos & $5-9$ anos & $>9$ anos & Total \\
$\begin{array}{l}\text { Bronquiolite Aguda (J21) } \\
\quad \text { Causada por vírus sincicial }\end{array}$ & $\mathbf{2 7 0}$ & $\mathbf{4 9}$ & $\mathbf{4}$ & $\mathbf{3}$ & $\mathbf{3 2 6}$ \\
$\begin{array}{l}\text { respiratório (J21.0) } \\
\quad \text { Casada por outros microorga- }\end{array}$ & 211 & 39 & 4 & 1 & 255 \\
$\begin{array}{l}\text { nismos (J21.8) } \\
\quad \begin{array}{l}\text { Sem agente etiológico identifi- } \\
\text { cado (J21.9) }\end{array}\end{array}$ & 31 & 9 & 0 & 2 & 42 \\
\hline
\end{tabular}

Fonte: DATASUS

\section{DISCUSSÃO}

Através da análise dos dados levantados, percebe-se que a maior percentagem (83,59\%) de internações devido à bronquiolite aguda ocorreu em indivíduos menores de 1 ano. Caracterizando esta faixa etária como um importante fator de risco para internações por bronquiolite.

Conforme a discriminação do DATASUS, a bronquiolite está disposta em três códigos do CID10, correspondendo a J21: Bronquiolite aguda; J21.0: Bronquiolite aguda devido ao vírus sincicial respiratório; J21.8: Bronquiolite aguda devido a outro microrganismo específico; J21.9: Bronquite aguda não-especificada. Apesar de a literatura apontar o vírus sincicial respiratório como principal agente viral dessa doença( ${ }^{(6)}$, os dados encontrados apontam apenas 29 internações atribuídas a ele, correspondendo a 8,89\%. A maior causa de internações está relacionada ao CID J21.8: Bronquiolite aguda devido a outro microrganismo específico $(78,22 \%)$, sugerindo, então, que pode haver uma escusa do diagnóstico etiológico. Uma vez que a bronquiolite é uma síndrome respiratória aguda, que se apresenta de maneira similar apesar de diversas etiologias virais (excetuando-se casos de bronquiolite bacteriana), e considerando que a classificação não muda o diagnóstico e a conduta, não se faz necessária a distinção do agente viral.
Dentre as causas de internações por bronquiolite aguda, as quais incluem crianças em mau estado geral, desidratadas, com esforço respiratório moderado a severo e hipoxêmicas, podemos citar a "internação social". Considera-se "internação social" como uma hospitalização devido a dificuldades econômicas e sociais do paciente, que é internado para ter acesso ao tratamento adequado ${ }^{(9)}$. Nesse contexto figuram situações em que a criança é admitida ao hospital por falta de condições, dos pais ou responsáveis, em oferecer cuidados domiciliares adequados, ou moradias de difícil acesso a serviços de saúde em tempo hábil.

Além disso, certas variáveis - como políticas de admissão dos centros, variabilidade da prática hospitalar, bem como as características do próprio paciente - podem interferir nos indicadores relacionados às taxas de internações sensíveis à atenção primária ${ }^{(1,10)}$.

\section{CONCLUSÃO}

A bronquiolite é uma doença que gera um alto índice de internações principalmente em indivíduos de faixa etária abaixo de um ano de idade. Como uma causa de internação sensível à atenção básica, essa injúria à saúde poderia ser prevenida através de uma atenção primária contínua e sistematizada que integre ações curativas e preventivas.

Nesse sentido, reforça-se a necessidade de fortalecimento das políticas públicas na atenção básica, já que desse modo, os agravos à saúde sensíveis à atenção básica poderiam ser evitados, otimizando a utilização dos demais níveis e auxiliando no bem estar da população.

Outro ponto relevante apontado por este estudo é a possível má classificação etiológica realizada nos serviços, uma vez que a causa mais frequente encontrada nos registros nacionais é a bronquiolite por outros microrganismos que não o vírus sincicial respiratório, como não temos plausibilidade biologia para supor que o Brasil tenha etiologia diferente para esta doença que o resto do mundo, parece-nos claro que medidas que visem melhorar qualidade dos registros sejam de fundamental importância, levando a uma consequente melhor planejamento das ações de saúde voltadas a esta doença.

\section{REFERÊNCIAS}

1. Starfield B. Atenção Primária: equilíbrio entre necessidades, serviços e tecnologias. Unesco, Edição BRASILEIRA 2002.

2. Brasil. Portaria No 221. 17 DE Abril. 2008. 
3. Nedel FB, Facchini la, Martim M, et al. Primary Health Care Risk Factors for Hospitalization for Ambulatory Care Sensitive Conditions: Systematic literature Review. Epidemiol. Serv. Saúde, Mar. 2010, vol.19, NO.1, P.61-75. ISSN 1679-4974.

4. Carvalho WB; johnston, Cíntia; FOnSeCA, Marcelo Cunio. Bronquiolite aguda, uma revisão atualizada. Rev. Assoc. Med. Bras., São Paulo, v. 53, n. 2, Apr. 2007.

5. Piedra PA, Stark AR. Bronchiolitis in infants and children: Clinical features and diagnosis. UpToDate.

6. Barr FE, Graham BS. Respiratory syncytial virus infection: Clinical features and diagnosis. UptoDate.

7. Piedra, Pedro A, Stark AR. Bronchiolitis in infants and children: Treatment; outcome; and prevention. UpToDate.

8. Organização Mundial da Saúde. Classificação estatística internacional de doenças e problemas relacionados À saúde. $10^{a}$ Revisão. São Paulo: Centro Colaborador da OMS para a Classificação de Doenças em Português, 1995.

9. Garbinato LR, et al. Prevalência de internação hospitalar e fatores associados: um estudo de base populacional em um centro urbano no Sul do Brasil. Cad. Saúde Pública vol.23 no.1 Rio de Janeiro Jan. 2007.

10. Notas Técnicas Sobre o Indicador - Internações por Condições Sensíveis à Atenção Primária - ICSAP. DisPoníVEL 